\title{
Exact inbreeding coefficient of maize synthetic varieties derived from mixed lines and single crosses
}

Crop Breeding and Applied Biotechnology 20(4): e31392047, 2020 Brazilian Society of Plant Breeding. Printed in Brazil http://dx.doi.org/10.1590/198470332020v20n4a60

\section{Denise Arellano-Suarez ${ }^{1}$, Juan Enrique Rodríguez-Pérez ${ }^{1}$, Aureliano Peña-Lomelí ${ }^{1}$ and Jaime Sahagún-Castellanos ${ }^{1^{*}}$}

\begin{abstract}
There is an apparent overvaluation in the inbreeding coefficient (IC) formula for a synthetic variety (SV) derived from a mixture of $S$ single crosses and L lines (FSyn ${ }_{L S C}$ ) in Zea mays. Therefore, our goal was to derive the exact and general FSyn ${ }_{L, S C^{\circ}}$ For the development of this $S V\left(S y n_{L, S C}\right)$, an even number of $L+2 S$ unrelated lines whose IC was $F(0 \leq F \leq 1)$ was considered. We show that the exact and general formula for FSyn ${ }_{L, S C}$ is: $F S y n_{L, S C}=(2 L+S)(1+F) /\left[4(L+S)^{2}\right]$, and that the ICs and genotypic means of two additional synthetics developed from the same lines (one derived from $(L+S 2) / 2$ single crosses and the other derived from the $L+2 S$ lines) must be equal. Among these three SVS, Syn ${ }_{L, S C}$ shows the largest IC.
\end{abstract}

Keywords: Zea mays L., random mating, identical by descent genes, coancestry, genotypic mean.

\section{INTRODUCTION}

In a finite population whose individuals reproduce by panmixia, mating between relatives will likely occur in subsequent generations, resulting in a reduction of vigor (Falconer and Mackay 2001). For this reason, formulae have been derived to calculate inbreeding coefficients (ICs) and to predict the performance of a crop variety called a synthetic variety (SV), or simply a synthetic.

Synthetic varieties of some cultivated species such as maize (Zea mays L.) are usually made with lines. However, they can also be generated with single crosses, three-way line crosses, double crosses, and mixtures of these hybrids (Márquez-Sánchez 2011). Each resulting synthetic will have a particular genotypic array, IC, and genotypic mean.

The mating systems among individuals are classified into two types: preferential and random or panmictic. In this paper, inbreeding (mating among related individuals) in the context of random mating will be addressed, because it involves the reproduction of synthetics. In an applied context, SV inbreeding is important because it is related to the genotypic mean of traits of economic importance, such as maize grain yield (Busbice 1970) and onion (Allium cepa L.) seed production (Magalhães et al. 2020)

Malécot (1948) defined the inbreeding coefficient (IC) as the probability that two genes in the same locus are identical by descent. Two genes are
*Corresponding author: E-mail: jsahagunc@yahoo.com.mx (iD) ORCID: 0000-0003-0965-9672

Received: 22 September 2019 Accepted: 04 September 2020 Published: 30 October 2020

${ }^{1}$ Universidad Autónoma Chapingo, Departamento de Fitotecnia, Carretera MéxicoTexcoco, 56.230, Chapingo, Estado de México, México 
considered identical by descent when both are copies of the same ancestral gene. In this context, the base population is the ancestor generation, which has no genes identical by descent and an IC equal to zero.

Cultivated species that mate randomly, such as onion and maize, facilitate the formation of synthetic varieties derived from several parents, each represented by $m$ plants. According to Wricke and Weber (1986), synthetics show genetic variability, adaptability to diverse environments, and stability in their corresponding genotypic arrays across generations. At the same time, randomness, a finite number of individuals (particularly when $m$ is small), and genetic variability among the plants representing each parent might cause inbreeding and genetic erosion.

Several recent studies have been conducted to investigate the adaptability and performance of synthetic varieties. Farid et al. (2019) found two adaptive synthetics whose grain yield under drought conditions was at least $6.0 \mathrm{t} \mathrm{ha}^{-1}$. Badu-Apraku et al. (2018) reported a genetic gain of $423 \mathrm{~kg} \mathrm{ha}^{-1} \mathrm{cycle}^{-1}$ in a SV after five cycles of $\mathrm{S}_{1}$ family selection for resistance to Striga hermonthica (Delile) Benth. This synthetic underwent three selection cycles for grain yield under drought. The International Maize and Wheat Improvement Center (CIMMYT) releases "open pollinated varieties" for grain yield potential and biotic and abiotic stress tolerance for areas prone to environmental stresses (Masuka et al. 2017). These varieties are synthetics whose parent lines that are a byproduct of the hybrid parental line development pipeline.

Maize synthetics, besides being used as improved varieties in developing countries, are also being used in breeding programs (Saboor et al. 2018). For instance, Oliveira et al. (2016) studied the methods to identify genotypes or populations that will ensure success when crossed. The authors used a diallel whose parents were eight synthetic varieties to study combining abilities and best linear unbiased predictions (BLUP). Articles on other methods to estimate combining abilities of potential parents of a SV or a hybrid have also been published. Genet et al. (2017) used testers and Afekhai et al. (2017) based this estimation on the use of the North Carolina mating design II. Furthermore, recurrent selection has also been used to improve performance of SVs (Baktash 2016) and Saito et al. (2018) considering adaptability and stability, found five corn lines suitable to produce dent synthetics resistant to gray leaf spot and northern leaf blight.

An economic advantage in using a SV is that it allows farmers to produce their own seed without changing the genotypic array, if there is no pollen contamination (Masuka et al. 2017). Furthermore, using hybrids instead of synthetic parents as lines can save resources (Márquez-Sánchez 2011). It might be assumed that a synthetic derived from hybrids is the same as the one that would be developed from the parental lines of such hybrids. However, this might not necessarily be the case. With hybrids, the demand for resources must be less. For example, with 16 lines it is possible to develop $\left(\begin{array}{c}16 \\ 2\end{array}\right),\left(\begin{array}{c}16 \\ 3\end{array}\right), \ldots,\left(\begin{array}{c}16 \\ 16\end{array}\right)$ synthetic varieties whose parents are $2,3, \ldots, 16$ lines, respectively, and the grain yields of the possible $\sum_{i=2}^{16}\left(\begin{array}{c}16 \\ i\end{array}\right)=65519$ synthetics can be predicted based on data obtained from evaluating the complete $16 \times 16$ diallel that would be formed with the 16 lines. Furthermore, with 8 single crosses involving the 16 lines, predicting the $\sum_{i=2}^{8}\left(\begin{array}{l}8 \\ i\end{array}\right)=$ 247 possible synthetics from two or more parents (single crosses) can be made based on the resulting $8 \times 8$ diallel. This is $75 \%$ smaller than the $16 \times 16$ diallel. With 4 double crosses involving the 16 lines, synthetic prediction can be made, but only for 15 synthetics from two or more double crosses. Moreover, many of the best synthetics might not be predicted in this manner. Therefore, other alternatives must be explored.

Some resources would be saved by using a synthetic whose parents are a mixture of a number of $S$ single crosses and $L$ lines $\left(S y n_{L S C}\right)$ instead of using only parental lines. In addition, if $L>0$ and $S>0$, more synthetics could be predicted than when their parents are only single crosses or double crosses. Sahagún-Castellanos et al. (2013) developed a formula for the IC of a Syn ${ }_{L, S C^{\circ}}$ In this case, the IC of all involved lines was called $F(0 \leq F \leq 1)$. However, this formula contains an apparent overestimation of the coancestry among individuals representing each single cross. This overvaluation would affect both the synthetic IC that would be formed with only single crosses $\left(F S y n_{S C}\right)$ and the IC of $S_{L y} n_{L S C}\left(F S y n_{L, S C}\right)$. The hypothesis of this study is that the intraparental coancestry was not derived accurately in previous studies. The main objective was to derive the exact and general IC (lines not necessarily pure) for synthetic varieties whose parents are $L$ lines and $S$ single crosses.

\section{MATERIAL AND METHODS}

The study was based on the one locus model of a population of a diploid species where its individuals mate randomly. To develop a SV whose parents are $L$ and $S$ single crosses, an even number of $L+2 S$ unrelated initial lines whose IC was 


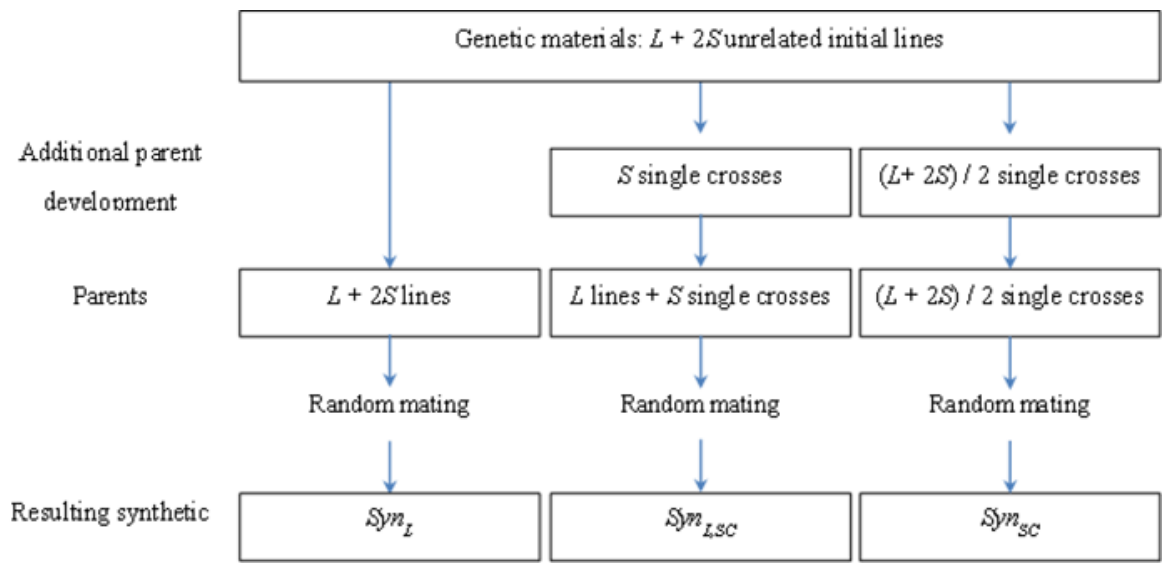

Figure 1. Development of three maize synthetic varieties $\left(S y n_{L^{\prime}} S y n_{L, S C^{\prime}}\right.$ and $\left.S y n_{S C}\right)$. Their parents were developed from an even number of $L+2 S$ unrelated lines. The three synthetics were obtained by randomly mating their respective parents.

$F(0 \leq F \leq 1)$ were considered. Then, $S$ single crosses were visualized with $2 S$ lines. Two additional synthetic varieties developed from the same $L+2 S$ lines were also visualized. Their parents were: 1 ) the initial $L+2 S$ lines $\left(S y n_{L}\right)$, and 2) $(L+2 S) / 2$ single crosses developed from all $L+2 S$ lines $\left(S y n_{s c}\right)$. In the three cases, each parent (line or single cross) was represented by $m$ plants (Figure 1 ).

The IC of $S y n_{L, S C}$ synthetic developed by the random pairing of $L$ lines and $S$ single crosses was determined based on Malécot (1948)'s approach using the concepts of genotypic array and the gametic array of a panmictic population. Kempthorne (1969) considers that if the frequency of the $A_{i}$ gene $(i=1,2 \ldots, a)$ in a panmictic population is $p_{i}$, the gametic $(G A A)$ and genotypic (GEA) arrays are expressed as:

$$
G A A=\sum_{i=1}^{a} p_{i} A_{i} \text { and } G E A=\sum_{i=1}^{a} \sum_{j=1}^{a} p_{i} p_{j} A_{i} A_{j}
$$

Classical probability was used to determine the IC of a SV. We assumed the existence of equally possible and mutually exclusive events, and that, the IC is the probability that two random genes, carried by two separate gametes (one male and one female) are identical by descent. $F S y n_{L, S C}$ is composed of contributions from two sources: self-fertilizations and intraparental crosses. To calculate the contribution of a source, the probability of identity by descent is multiplied by the quotient of the number of the corresponding genotypes in the genotypic array divided by the total genotype number. Thus, the IC of the synthetic is the sum of both contributions. This is equivalent to what occurs in the GEA when $A A_{j}$ is replaced by $\mathrm{P}\left(A_{i} \equiv A_{j}\right)$, i.e. the probability that $A_{i}$ and $A_{j}$ are identical by descent.

The random mating of the $m(L+S)$ plants that generate the $S y n_{L, S C}$ synthetic implies random mating among the $m$ plants of each parent and any other parental group. Therefore, the derivation of the FSyn ${ }_{L, S C}$ formula was based on the inbreeding contributions of each line and each single cross.

Regarding the IC of the progeny of a single cross, the two parental lines were assumed as two virtual populations represented by $A_{1} A_{2}$ and $B_{1} B_{2}$, such that $\mathrm{P}\left(A_{1} \equiv A_{2}\right)=P\left(B_{1} \equiv B_{2}\right)=F$.

\section{RESULTS AND DISCUSSION}

The single cross $A_{1} A_{2} \times B_{1} B_{2}$ produces progeny whose genotypic array $\left(G E A_{S C}\right)$ is:

$$
G E A_{S C}=\frac{1}{4} A_{1} B_{1}+\frac{1}{4} A_{1} B_{2}+\frac{1}{4} A_{2} B_{1}+\frac{1}{4} A_{2} B_{2}
$$

The set of $m$ plants that represents this single cross can be visualized as the result of taking a random sample (with replacement) of size $m$ from a population formed by the four genotypes of this $G E A_{S C}$ (Equation 1 ). The random 
pairing of these $m$ plants produces progeny whose genotypic array $\left(G E A_{P S C}\right)$ is the product of the gametic array $\left(G A A_{s C}\right)$ "multiplied" by itself. Since

$$
G A A_{S C}=\frac{1}{4} A_{1}+\frac{1}{4} A_{2}+\frac{1}{4} B_{1}+\frac{1}{4} B_{2}
$$

Then, the GEA $A_{P S C}$ is:

$$
\begin{gathered}
G E A_{P S C}=\frac{1}{16} A_{1} A_{1}+\frac{1}{16} A_{2} A_{2}+\frac{1}{16} B_{1} B_{1}+\frac{1}{16} B_{2} B_{2}+\frac{2}{16} A_{1} A_{2}+\frac{2}{16} B_{1} B_{2}+\frac{2}{16} A_{1} B_{1}+ \\
\frac{2}{16} A_{1} B_{2}+\frac{2}{16} A_{2} B_{1}+\frac{2}{16} A_{2} B_{2}
\end{gathered}
$$

The IC of the GEA $A_{P S C}\left(F_{S C}\right)$ of Equation 3 is formed only from the genotypes where both genes have the same letter (because the lines are not related). Thus, according to the IC definition, based on Equation 3

$$
\begin{aligned}
F_{S C}=\frac{1}{16}+\frac{1}{16} & +\frac{1}{16}+\frac{1}{16}+\frac{2}{16} F+\frac{2}{16} F \\
& =\frac{4}{16}(1+F) \\
& =\frac{1+F}{4}
\end{aligned}
$$

The inbreeding coefficient of the population produced by the random mating of $m$ plants representing a single cross $\left(F_{s C(m)}\right)$ can also be expressed in terms of $m$ and the two inbreeding sources: the $m$ self-fertilizations and the $m(m-1)$ intraparental crosses, whose average inbreeding coefficients are $1 / 2$ and $\Gamma_{0, w}$ (the average coancestry among individuals within a parent), respectively. In addition: 1 ) each self-fertilization and each cross produces progeny whose genotypes can be visualized as the 4 possible outcomes that result from uniting one random male and female gene, and 2 ) the total number of possible results from the random mating of $m$ plants is $4 m+4 m(m-1)=4 m^{2}$. Thus, based on these considerations and the definition of the inbreeding coefficient:

$$
\begin{array}{r}
F_{S C(m)}=\frac{4 m\left(\frac{1}{2}\right)}{4 m^{2}}+\frac{4 m(m-1) \Gamma_{O, W}}{4 m^{2}} \\
=\frac{4 m\left(\frac{1}{2}\right)+4 m(m-1) \Gamma_{O, W}}{4 m^{2}} \\
=\frac{4 m\left(\frac{1}{2}\right)+4 m(m-1) \Gamma_{O, W}}{(2 m)^{2}}
\end{array}
$$

Since $F_{s C}=(1+F) / 4($ Equation 4$)$ and $F_{s C}=F_{s C(m)}($ Equation 5):

Then, solving for $\Gamma_{0, w}$ :

$$
\frac{1+F}{4}=\frac{4 m\left(\frac{1}{2}\right)+4 m(m-1) \Gamma_{o, W}}{(2 m)^{2}}
$$

$$
\Gamma_{O, W}=\frac{m(1+F)-2}{4(m-1)}
$$

It is evident that $\Gamma_{0, w}$ depends directly on $F$. Regarding the relationship between $\Gamma_{0, w}$ and $F$, consider that the cross of two individuals produces progeny whose inbreeding coefficient is directly related to the level of coancestry of these two individuals. In a panmictic population, the inbreeding level of the progeny and the coancestry of their parents are equal.

By substituting the result of Equation 6 into Equation 5, we obtain:

$$
F_{S C(m)}=\frac{4 m\left(\frac{1}{2}\right)+4 m(m-1)\left[\frac{m(1+F)-2}{4(m-1)}\right]}{(2 m)^{2}}
$$


The $F_{s c(m)}$ proportion due to self-fertilization $\left[F_{s c, x}\right]$ according to Equation 7 is:

$$
F_{S C, X}=\frac{4 m\left(\frac{1}{2}\right)}{(2 m)^{2}}
$$

This reduces to the following equation:

$$
F_{S C, X}=\frac{1}{2 m}
$$

According to Equation (9), there is an inverse relationship between $F_{s c, x}$ and $m$. That is, when $m$ increases, the importance of self-fertilization as an inbreeding source decreases. Notably, $F_{s c, x}$ does not depend on the inbreeding coefficient of the lines. However, the self-pollination of a plant formed by the cross between two unrelated lines of any inbreeding level will always produce progeny whose expected genotype frequency of two identical genes is $1 / 2$. Regarding the coancestry among individuals within a parent, the contribution to $F_{S C(m)}\left(F_{s c, \Gamma_{0, \mathrm{~W}}}\right)$, based on Equations 5 and 6 , is:

$$
\begin{gathered}
F_{S C, \Gamma_{O, W}}=\frac{4 m(m-1)\left[\frac{m(1+F)-2}{4(m-1)}\right]}{(2 m)^{2}} \\
=\frac{m(1+F)-2}{4 m} \quad(10)
\end{gathered}
$$

As expected, according to Equation $10, F_{s c, \Gamma_{0, \mathrm{~W}}}$ is directly related to $F$ and $m$. If the initial lines were pure $(F=1)$, then $F_{s c, \Gamma_{0, w}}=(m-1) / 2 m$. Thus, $F_{s c, \Gamma, \mathrm{w}}$ only depends directly on $m$. Note that for this particular case, $F_{s c, \Gamma_{0, \mathrm{w}}}$ is greater than $F_{s c, \mathrm{x}}$ only when $m>2$.

An equation for $F S y n_{S C}$ was previously derived (Márquez-Sánchez 2011). This equation, however, is restricted to the case of pure lines $(F=1)$. The obtained results are coincident with our results for a similar case. For the general case where $0 \leq F \leq 1$, Sahagún-Castellanos et al. (2013) considered that $\Gamma_{0, W}=(1+F) / 2$, which is not possible for a population formed by $m$ representatives of a single cross between two unrelated lines. In this case, the maximum value for a coancestry is $1 / 2$, which is the coancestry of a plant with itself (via self-fertilization) or between two individuals that have the same genotype.

As previously stated, the genotypic mean of a SV trait such as grain yield in maize has an inverse relationship with inbreeding (Busbice 1970). According to Equation 9, for the case of inbreeding by self-fertilization in a synthetic developed from single crosses, as $m$ increases, the genotypic mean increases because the IC decreases. An inverse effect of the same magnitude is observed in $F_{s c, \Gamma \text {, }}$ (Equation 10), since it can be expressed as $(1+F) / 4-(1 / 2 m)$. This implies that the expected genotypic mean does not depend on $m$. Consider now the random pairing of the $m S$ representatives of the $S$ single crosses. The resulting synthetic $\left(S y n_{s C}\right)$ is composed of the progeny of $S(S-1)$ crosses between parents and the intraparental crosses within each parent. Since the inbreeding coefficients of these two crosses are zero (because the single crosses are unrelated) and $F S y n_{S C(m)}$ (Equation 7), respectively, then the $S_{y n_{S C}} I C\left(F S y n_{s C}\right)$ is equal to:

$$
\begin{gathered}
\text { FSyn }_{S C}=\frac{S(S-1)(0)+S F_{S C(m)}}{S^{2}} \\
=\frac{F_{S C(m)}}{S} \\
=\frac{4 m\left(\frac{1}{2}\right)+4 m(m-1)\left[\frac{m(1+F)-2}{4(m-1)}\right]}{S(2 m)^{2}}
\end{gathered}
$$

Or, in compact form (Equation 4):

$$
\text { FSyn }_{S C}=\frac{1+F}{4 S}
$$

The synthetic developed by randomly mating $L$ lines, each represented by $m$ plants $\left(S y n_{L}\right)$, is a population whose IC 
is composed of the contribution from self-fertilization and from intraparental coancestry. To derive the IC for $\operatorname{Syn}_{L}\left(\right.$ FSyn $\left.n_{L}\right)$ , consider that if the $A_{1} A_{2}$ genotype represents the line, $A_{1} A_{2}$ self-fertilization produces the genotypic array (1/4) $A_{1} A_{1}$ $+(1 / 2) A_{1} A_{2}+(1 / 4) A_{2} A_{2}$, whose IC is $(1 / 4)(1)+(1 / 2)(F)+(1 / 4)(1)=(1+F) / 2$ Furthermore, an intraparental cross must also produce the genotypic array $(1 / 4) A_{1} A_{1}+(1 / 2) A_{1} A_{2}+(1 / 4) A_{2} A_{2}$ whose IC is also $(1 / 4)(1)+(1 / 2)(F)+(1 / 4)(1)=(1+$ F)/2. Therefore:

$$
\text { FSyn }_{L}=\frac{4 L m\left(\frac{1+F}{2}\right)+4 L m(m-1)\left(\frac{1+F}{2}\right)}{(2 m L)^{2}}
$$

Or, in reduced form:

$$
\text { FSyn }_{L}=\frac{1+F}{2 L}
$$

In addition, it is evident that the contribution from self-fertilizations $\left(F_{L, X}\right)$ to $F S_{L} n_{L}$ is:

$$
F_{L, X}=\frac{1}{L m}\left(\frac{1+F}{2}\right)
$$

Equation 14 shows that $F_{L, X}$ depends directly on $F$ and inversely on $L$ and $m$. Since $0 \leq F \leq 1$, as $L$ and $m$ increase, $F_{L, X}$ decreases.

The contribution by intraparental coancestry to $F S_{L}\left(F_{S C, \Gamma_{0, W}}\right)$ is:

$$
\begin{gathered}
F_{L, \Gamma_{O, W}}=\frac{(m-1)}{L m}\left(\frac{1+F}{2}\right) \\
=\frac{1+F}{2 L}-\frac{1}{L m}\left(\frac{1+F}{2}\right)
\end{gathered}
$$

Equations 14 and 15 show that contributions by self-pollination and intraparental coancestry are counteracted by changes in $\mathrm{m}$; that is, $\mathrm{m}$ does not affect $F \mathrm{Syn}_{L^{\prime}}$ as evidenced by its reduced formula (Equation 13).

If the parents of a synthetic are $L$ lines and $S$ single crosses $\left(S y n_{L, S C}\right)$, the IC of this synthetic $\left(F S y n_{L, S C}\right)$ must be composed of the contribution from self-pollination and from intrapaternal crosses from both lines and single crosses (four terms). Based on Equations 11 and 13:

$$
\begin{gathered}
\text { FSyn }_{L, S C}=\frac{4 m L\left(\frac{1+F}{2}\right)+}{4 L m(m-1)\left(\frac{1+F}{2}\right)+4 m S\left(\frac{1}{2}\right)+4 S m(m-1)\left[\frac{m(1+F)-2}{4(m-1)}\right]} \\
=\frac{4 m^{2} L\left(\frac{1+F}{2}\right)+2 m S+S m[m(1+F)-2]}{4 m^{2}(L+S)^{2}} \\
=\frac{4 m^{2} L\left(\frac{1+F}{2}\right)+S m[m(1+F)]}{4 m^{2}(L+S)^{2}} \\
=\frac{4 m^{2} L\left(\frac{1+F}{2}\right)+4 m^{2} S\left(\frac{1+F}{4}\right)}{4 m^{2}(L+S)^{2}} \\
=\frac{L\left(\frac{1+F}{2}\right)}{(L+S)^{2}}+\frac{S\left(\frac{1+F}{4}\right)}{(L+S)^{2}}
\end{gathered}
$$

Or in reduced form:

$$
F \text { Syn }_{L, S C}=\frac{(2 L+S)(1+F)}{4(L+S)^{2}}
$$

This is the exact and general $\operatorname{Syn}_{L, S C}(0 \leq F \leq 1) \mathrm{IC}$.

From Equation 17: 
a) If $S=0, F_{S y n}, S C=F S y n_{L}$. Therefore, the IC of a synthetic whose parents are $L$ lines is:

$$
\text { FSyn }_{L}=\frac{1+F}{2 L}
$$

b) If $L=0, F_{S y n}=F S y n_{S C}$. Thus, the IC of a synthetic whose parents are $S$ single crosses is:

$$
F S y n_{S C}=\frac{1+F}{4 S}
$$

Rodríguez-Pérez et al. (2019) derived the IC of a synthetic developed from the random mating of $d$ double crosses, each represented by a "large" number of plants $\left(F S y n_{D C}\right)$. They found that from the ICs for Syn (Equation 18) and for $S y n_{S C}$ (Equation 19), $F S y n_{D C}=(1+F) / 8 d$, as expected.

The independence between the inbreeding coefficients of the synthetic varieties and $m$ in Equations 17 to 19 occurs because the gene frequencies are not altered when $m$ is increased or decreased. Therefore, neither the synthetic genotypic arrays, nor their inbreeding coefficients or genotypic means should be modified.

Consider now that if the initial lines were pure $(F=1)$, Equation 17 reduces to:

$$
\text { FSyn }_{L, S C}=\frac{2 L+S}{2(L+S)^{2}}
$$

Equations 18 and 19 were generated directly from Equations 12 and 13 . These equations imply that for one synthetic formed with $L^{\prime}=L+2 S$ lines and another with $S^{\prime}=L^{\prime} / 2$ single crosses which involve the same $L^{\prime}$ lines, their inbreeding coefficients $\left(F S y n_{L^{\prime}}\right.$ and $\left.F S y n_{s C^{\prime}}\right)$ must be equal: $F S y n_{L^{\prime}}=F S y n_{S C^{\prime}}$. This occurs because the gene frequencies involved in the two parental sets are the same in both cases. If from all these $L+2 S$ lines, a $S y n_{L, S C}$ with $S \geq 1$ and $L \geq 1$ is generated, the gene frequencies are unbalanced and $F S y n_{L, S C}$ must be larger than $F S y n_{L^{\prime}}$ and $F S y n_{S C^{\prime}}$. Similarly, Ibarra-Sánchez et al. (2019) found that the IC of a synthetic developed from $t=L^{\prime} / 3$ three-way line hybrids $\left(S y n_{T}\right)$ is larger than $F S y n_{L^{\prime}}$ and $F S y n_{S C^{\prime}}$.

Regarding the number of parents, the inverse FSyn $n_{L}$ and $F S y n_{S C}$ relationship (Table 1 and Equations 17-19) occurs because each increase in the number of parents increases the number of interparental crosses (which do not contribute to inbreeding) in a greater proportion than the number of self-pollinations and intrapaternal crosses, which provide genotypes formed by two identical by descent genes.

The direct relationship of $F$ with the IC of the synthetics evidenced in Equations 17 to 19 is obvious. When $F$ increases, the contributions to the inbreeding of the synthetic by self-fertilization and intraparental crosses also increase, while the interparental crosses will never contribute to the IC because the parents are not related. Furthermore:

a) From Equation 18, if $F=1$, for a $S_{y} n_{L}$ formed with $L$ lines:

$$
\text { FSyn }_{L}=\frac{1}{L}
$$

b) Similarly, according to Equation 19 , if $F=1$ for a $S y n_{s c}$ developed from $S$ single crosses:

$$
F S y n_{S C}=\frac{1}{2 S}
$$

Equations 18, 21, and 22 have already been reported (Sahagún-Castellanos et al. 2013), but not Equations 17, 19, and 20.

Equations 17 to 22 are directly interpretable. Table 1 shows the inbreeding coefficients for 88 synthetic varieties formed with 22 combinations of lines and single crosses, each with four levels of inbreeding in the initial parental lines.

As expected from Equation 17, Table 1 shows that for each mixture of $L$ lines and $S$ single crosses, the inbreeding coefficients are directly and linearly related to $F$. However, in synthetic varieties developed with the same number of initial lines $(6,8,10$, or 12$)$, for each of four $F$ values $(0.00,0.50,0.75$ and 1.00$)$, the IC of the synthetic varieties formed only with lines or only with single crosses are equal. This occurs because the genes and their frequencies are the same in both cases. This homogeneity of gene frequencies and random mating maximizes the formation of genotypes containing 
Table 1. Inbreeding coefficients of synthetic varieties developed by the random pairing of a mixture of $L$ lines and $S$ single crosses. Four inbreeding coefficients $(F)$ of the initial parental lines $(0.00,0.50,0.75$ and 1.00$)$ were considered. Calculations were based on Equation 17

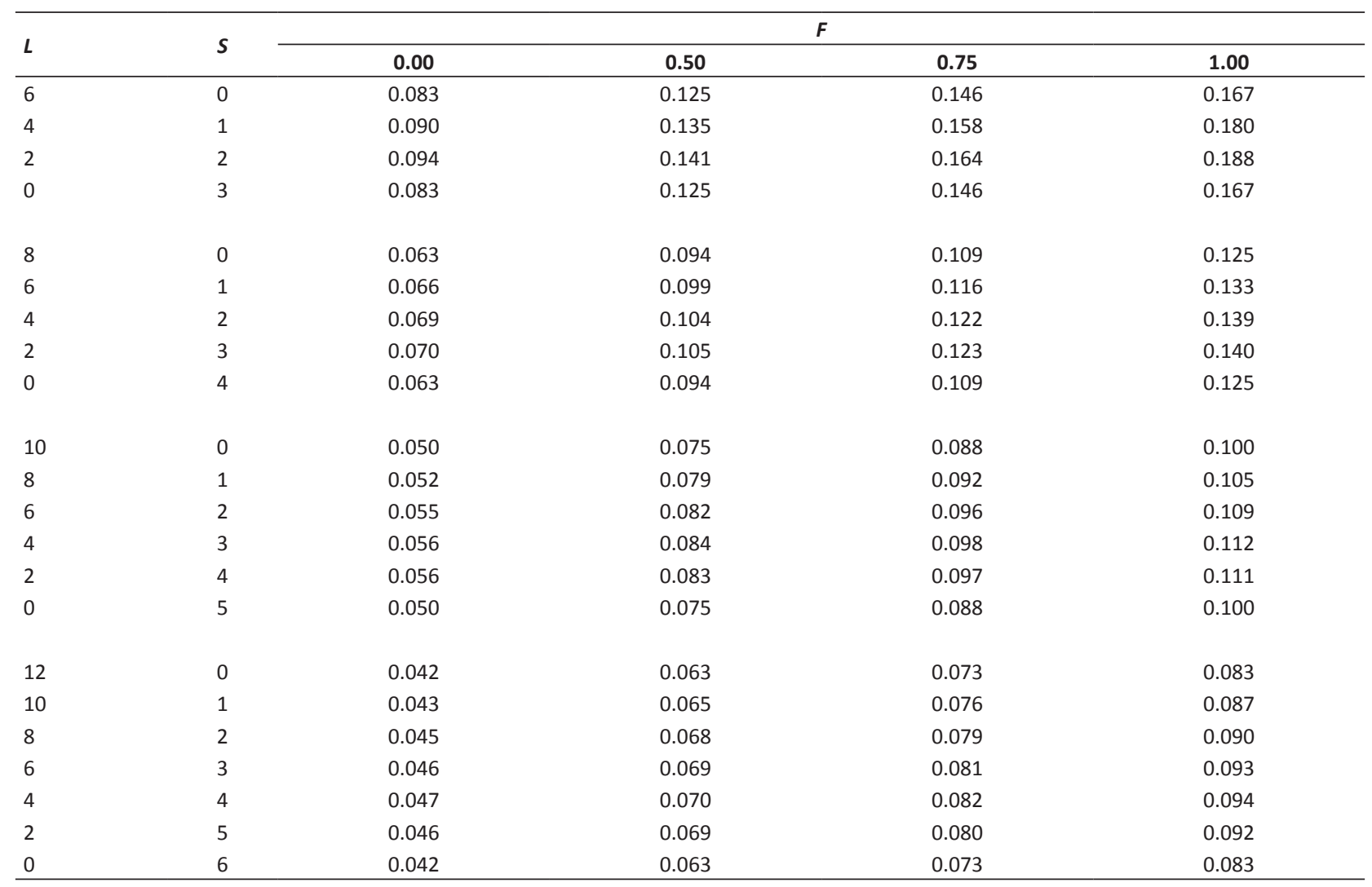

two genes from two different lines. However, as $L$ approaches $S$, the resulting inbreeding coefficients of the synthetic are larger because the variability of the gene frequencies of the $L+2 S$ lines involved in the development of $S y n_{L, S C}$ is increased. This larger gene frequency variance favors the increase of the frequency of genotypes formed by two identical by descent genes, which are the genes whose frequencies are the largest (those of the lines).

In summary, although the 3 synthetics have the same genes, the gene frequencies are the same only in $S y n_{s c}$ and $S y n_{L}$. Therefore, a) $S y n_{S C}=S y n_{L} \neq S y n_{L, S C}$, b) $F S y n_{S C}=F S y n_{L}<F S y n_{L, S C^{\prime}}$ and c) $S y n_{L, S C}$ must have the smallest genotypic mean (Busbice 1970).

\section{ACKNOWLEDGEMENTS}

We would like to thank Editage (www.editage.com) for English language editing.

\section{REFERENCES}

Afekhai A, Nwaoguala C and Alika J (2017) Estimation of combining ability for yield and component traits in maize (Zea mays L.) cultivars using North Carolina mating design II. Albanian Journal of Agriculture Science 16: 192-201

Badu-Apraku B, Ifie B, Talabi A, Obeng-Bio E and Asiedu R (2018) Genetic variances and heritabilities of traits of an early yellow maize population after cycles of improvement for Striga resistance and drought tolerance. Crop Science 58: 2261-2273.

Baktash F (2016) Modified mass selection within corn synthetic variety.

The Iraqi Journal of Agricultural Sciences 47: 391-395.

Busbice T (1970) Predicting yield of synthetic varieties. Crop Science 32: $271-274$.

Falconer DS and Mackay TFC (2001) Introducción a la genética cuantitativa. Longman Group Limited, Zaragoza, 490p. 
Exact inbreeding coefficient of maize synthetic varieties derived from mixed lines and single crosses

Farid M, Musa Y, Nasaruddin and I Ridwan (2019) Selection of various synthetic maize (Zea mays L.) genotypes on drought stress condition. Earth and Environmental Science 235: 1-6.

Genet Y, Tongoona P and Ifie B (2017) Classification of selected white tropical maize inbred lines into heterotic groups using yield combining ability effects. African Journal of Agricultural Research 12: 16741677.

Ibarra-Sánchez A, Rodríguez-Pérez J and Sahagún-Castellanos J (2019) General inbreeding coefficient of maize synthetics derived from three-way line hybrids. Terra Latinoamericana 37: 479-485.

Kempthorne O (1969) An introduction to genetic statistics. The lowa State University Press, Ames, 545p.

Malécot G (1948) Les mathématiques de I’hérédité. Masson et Cie, Paris, 66p.

Magalhães D, Mascarenhas G, Queiroz J, Freitas J, Oliveira A and Camargos $R$ (2020) Reduced inbreeding depression in separate generations of onions. Crop Breeding and Applied Biotechnology 20: 1-10.

Márquez-Sánchez F (2011) Maize synthetics from a mixture of single, three-way, and double cross hybrids using inbreeding coefficients and mean predictions. Maydica 56: 341-342.

Masuka B, Magorokosho C, Olsen M, Atlin G, Bänziger M, Pixley K, Vivek $B$, Labuschagne M, Matemba-Mutasa R, Burgueño J, Macrobert J, Prasanna B, Das B, Makumbi D, Tarekegne A, Crossa J, Zaman-Allah M, van Biljon A and Cairns J (2017) Gains in maize genetic improvement in Eastern and Southern Africa: II. CIMMYT open-pollinated variety breeding pipeline. Crop Science 57: 180-191.

Oliveira G, Buzinaro R, Revolti L, Giorgenon C, Charnai K, Resende D and Moro $G$ (2016) An accurate prediction of maize crosses using diallel analysis and best linear unbiased predictor (BLUP). Chilean Journal of Agricultural Research 76: 294-299

Rodríguez-Pérez J, Peña-Lomelí A, Villanueva-Verduzco C and SahagúnCastellanos J (2019) General and exact inbreeding coefficient of maize synthetics derived from double crosses. Agrociencia 53: 235-244.

Saboor A, Ullah H, Shahwar D, Fahad S, Khan N, Yasir M, Wahid F, Adnan $M$ and Noor M (2018) Heritability and correlation analysis of morphological and yield traits in maize. Journal of Plant Biology Crop Research 2: 1-8.

Sahagún-Castellanos J, Rodríguez-Pérez J and Escalante-González J (2013) Yield prediction and inbreeding of maize synthetics generated with lines and single crosses. Classic probability. Revista de la Facultad de Ciencias Agrícolas. Universidad Nacional de Cuyo 45: 75-84.

Saito B, Queiroz L, Costa JA and Goodman M (2018) Adaptability and stability of corn inbred lines regarding resistance to gray leaf spot and northern leaf blight. Crop Breeding and Applied Biotechnology 18: $148-158$.

Wricke G and Weber WE (1986) Quantitative genetics and selection in plant breeding. Walter de Gruyter, Berlin, 406p. 